\title{
RESEARCH ENERGY AND RESOURCE SAVING OPERATING MODES OF THE PUMP UNIT
}

\author{
Khushnud Sapaev ${ }^{l}$, Shukhrat Umarov ${ }^{l}$, Islombek Abdullabekov ${ }^{1}$ \\ ${ }^{1}$ Tashkent State Technical University, Department of Electromechanics and Electrotechnology, Tashkent, Uzbekistan
}

\begin{abstract}
The article presents the results of the study of operating modes of pumping stations as a set of all its elements. In this case, the water-lifting pumping station was considered as a class of turbomachines functioning in the systems of machine water-lifting. The development of new technical solutions for the creation of controlled systems of electric drives is proposed that most fully takes into account the specific features of the mutual influence of hydraulic and electromechanical modes and methods of influencing the technological process.
\end{abstract}

\section{Introduction}

Undoubtedly, one of the most promising areas of great scientific and practical importance is research aimed at achieving energy and resource conservation through the transition from an unregulated electric drive of energyintensive mechanisms for general industrial purposes to a regulated one, which provides the greatest effect in terms of energy saving and increasing a number of technological indicators. process $[1,2,10]$.

Therefore, the development and implementation of energy-saving modes of operation of variable multi-motor electric drives of pumping units, as well as the development of scientifically substantiated solutions for the construction of automatic control systems for pumping stations, taking into account the technological regime of water supply and rational use of water resources and specific energy costs is of important practical importance $[3,4,5]$.

\section{Influence of fluctuations in the water level in the intake structure of a pumping station on energy and resource-saving modes of operation of the pumping unit}

At irrigation water-lifting pumping stations (PS), during the seasonal period of their operation, there is a periodic change in one of the defining technological parameters as the lower level of the $\nabla_{\mathrm{L}}$ water pool in the intake structure of the PS [6]. The latter largely depend on the hydrogeological regimes of the functioning of the water source, the inlet and outlet canals of the irrigation water supply systems, as well as the regimes of water consumption of irrigated areas of agricultural crops.

The main hydraulic modes during their operation include: uniform, maintenance of the downstream water levels with overflowing volumes, which should not allow healing of canals and outlets of the PS, overflow of water through hydraulic structures in case of sudden forced stops of the pumping unit (PU), ensure without receipt work and the set mode of operation of the scientific equipment.

Let us consider the operating mode of the PU in conditions of a change in the level of the water horizon in the downstream of the PS, based on the well-known [6] graph-analytical method for constructing the operating characteristics of a centrifugal pump and a pressure head pipeline network.New designs of MFS have been developed [1,2]. It is shown that in a MFS containing coaxially located annular magnetic circuits with sections of measuring windings and through slots made along the circumference, dividing each annular magnetic circuit into two rods, elastic conical bushings located coaxially with the annular magnetic circuits and interacting with the bases with the surfaces of the annular magnetic circuits, performing ring magnetic cores are identical and the introduction of additional, concentrically and mutually mirrored elastic conical bushings, lips Installed with the possibility of simultaneous interaction with the inner and outer surfaces of the annular magnetic cores, as well as the inclusion of the measuring windings of the annular magnetic cores with each other in series, increases the sensitivity, accuracy and linearity of the sensor conversion characteristics. In addition, it has been shown that the implementation of through slots on the forming surfaces of the annular magnetic cores and the reinforcement of the horseshoe-shaped ring casings of inelastic material on the inner and outer forming surfaces of the annular magnetic cores, on which the bases of the conical elastic bushings act, increases the sensitivity of the MFS. 


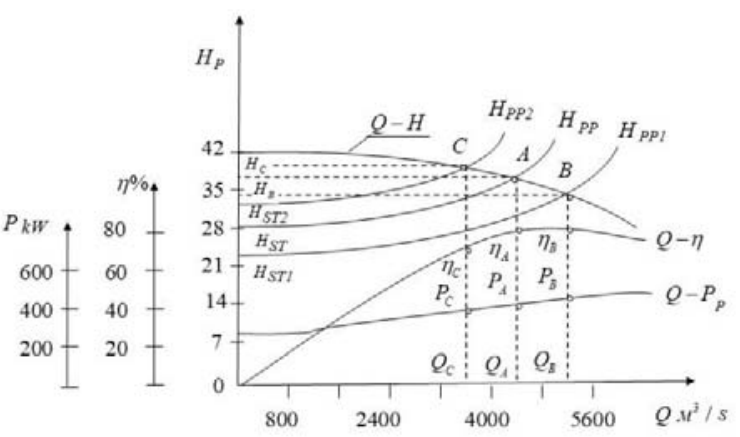

Fig. 1. Influence of changes in the water level in the downstream of the water intake construction of a pumping station for pumping unit mode

In (Fig. 1) shows the flow-pressure (Q-H), flow-power $\left(\mathrm{Q}-\mathrm{P}_{\mathrm{P}}\right)$ and flow-efficiency $(\mathrm{Q}-\eta)$ characteristics of the $5000 \mathrm{D}-32$ centrifugal pump, as well as the curve of the $\mathrm{H}_{\mathrm{PP}}$ pressure pipeline at a static head $\mathrm{H}_{\mathrm{SP}}$. The point $\mathrm{A}$ of the intersection of the Q-H characteristic of the centrifugal pump with the $\mathrm{H}_{\mathrm{PP}}$ curve corresponds to the steady-state operation of the "pump-pressure network" system with coordinates satisfying the required flow rate $\mathrm{Q}_{\mathrm{A}}$, the developed head $\mathrm{H}_{\mathrm{A}}$, power $\mathrm{P}_{\mathrm{MA}}$ and efficiency $\eta_{\mathrm{A}}$.

Periodic changes in the water level in the supply channel, and, consequently, in the water intake structure of the PS, as well as its changes in the outlet, affect the operating mode of the PU. It is obvious that the pressure losses in the pipeline (pressure network) of the PU, provided they are provided with an appropriate water supply, practically remain constant. Analysis of the equation for the characteristics of the pressure head network shows that $\mathrm{H}_{\mathrm{SP}}$ is the coordinate of the vertex of the quadratic parabola at supply $\mathrm{Q}=0$. This means that when the value of $\mathrm{H}_{\mathrm{SP}}$ changes, the coordinate of the top of the pressure network characteristic also changes by the amount of increase or decrease in the water level in the downstream of the PS.

Consequently, with an increase in the water level $\nabla_{\mathrm{L}}$ in the water intake structure of the PS, the static head will decrease $\mathrm{H}_{\mathrm{SP} 1}<\mathrm{H}_{\mathrm{SP}}$. In this case, the operating mode of the "pump - pressure network" system will be characterized by a new operating point $\mathrm{Bc}$ with coordinates $\mathrm{Q}_{\mathrm{B}}, \mathrm{H}_{\mathrm{B}}, \mathrm{P}_{\mathrm{PB}}, \eta_{\mathrm{B}}$, in which the pressure developed by the centrifugal pump decreases, the supply and power on the PU shaft increase, and the efficiency decreases. An increase in the water supply with an increase in the water horizon in the $\nabla_{\mathrm{L}}$ causes, in general, an increase in the productivity of the water supply, which causes inconsistencies in the schedules of water consumption and its coverage, leading to overconsumption of water resources with all the ensuing negative consequences. Other undesirable factors are a decrease in the efficiency of the PS, an increase in the power consumed by it, leading both to an overload of the drive electric motor and, in some cases, its heating above the permissible temperature, and to a decrease in the energy efficiency of the PS and, as a consequence, to an increase in the unit electricity costs.

With a decrease in the water level $\nabla_{\mathrm{L}}$ in the water intake structure of the PS, the static head will increase by the amount of the decrease in the water level, that is, $\mathrm{H}_{\mathrm{SP} 2}>\mathrm{H}_{\mathrm{SP}}$. In this case, the operating mode of the "pump - pressure network" system is characterized by another regime point $\mathrm{C}$, which has coordinates $\mathrm{Q}_{\mathrm{C}}, \mathrm{H}_{\mathrm{C}}, \mathrm{P}_{\mathrm{PC}}, \eta_{\mathrm{C}}$. Consequently, with a decrease in the water horizon $\nabla_{\mathrm{L}}$, the pressure developed by the centrifugal pump increases, the supply and the power consumed by it decrease, with a slight decrease in the value of its efficiency. A decrease in the PU supply leads to a deviation of the covering schedule of the PS water supply towards its underestimation. The operation of the PS, at this stage of the graph with the same number of PU, can contribute to a further decrease in the water level in the intake structure of the PS, which will subsequently cause the forced transfer of the PU to another regime point with a reduced flow, a deteriorated value of efficiency and an admissible suction head of the centrifugal pump.

In order to ensure the energy-efficient operation of the units of the water-lifting pumping station, there is also a need to stabilize the level of the pool of water both in its supply channel and, therefore, in the intake structure of the pumping station itself [7].

In modern domestic and foreign systems, regulation of operating modes of pumping units is carried out by means of an automated controlled electric drive. In such systems, the controlled parameter is the water head level in the intake structure of the pumping station, the value of which is selected based on the following considerations. From an energy point of view, the level of the water head in the intake structure of the pumping station should be maintained within strictly defined limits, which contributes to ensuring the minimum specific consumption of electrical energy of its pumping units [8]. However, in this case, one should take into account the real value of the incoming amount of water from the supply channel through its blocking structure, since the highly declared volume of water transported in it may not correspond to the supply realized by it by the pumping station units. This, in turn, can lead to an inevitable malfunction of the automatic control system, energyefficient stable maintenance of the water head level in the intake structure of the pumping station.

The scheme of stabilizing the water level in the intake structure of the pumping station is considered using the example of the system shown in (Fig. 2) and the process of its stabilization in (Fig. 3).

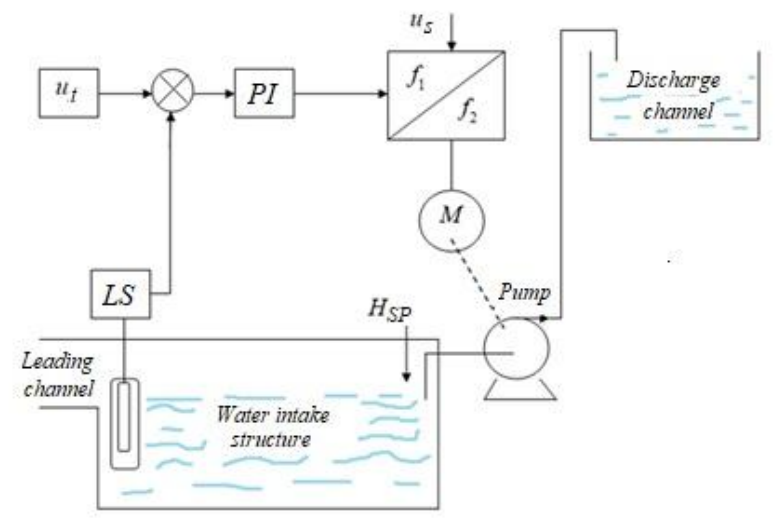

Fig. 2. Scheme of stabilization of the downstream water level in pumping station intake 

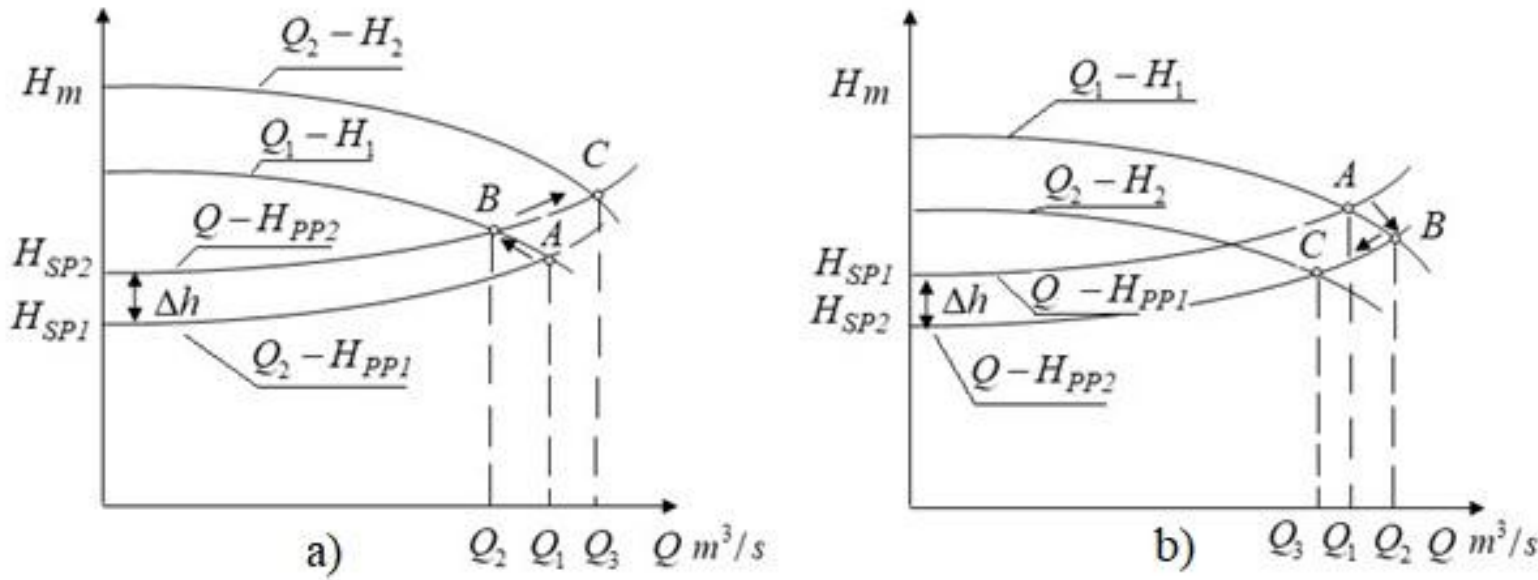

Fig. 3. Graphs of the processes of stabilization of the levels of water ponds in the intake structure of the pumping station: a) increase in water inflow by the value of $\Delta h$; b) decrease in water inflow by $\Delta h$.

It is known that at some point in time, as a result of an intense inflow of water through the supply channel of the PS, the level of the lower pool of water $\nabla_{\mathrm{L}}$ in its intake structure may increase by some value $\Delta \mathrm{h}$.

In this case, the static head $\mathrm{H}_{\mathrm{SP} 1}$ of the pumping station will decrease by the same value $\Delta h$ and will be equal $H_{S P 2}$ $=\mathrm{H}_{\mathrm{SP} 1}-\Delta \mathrm{h}$, where the water supply by the pumping unit becomes equal to $\mathrm{Q}_{2}$ (Fig. 3).

Suppose (Fig. 3) that at a static head $\mathrm{H}_{\mathrm{SP} 1}$, the PU operated at a steady state point $\mathrm{A}$, the intersection of the flow-pressure $\mathrm{Q}_{1}-\mathrm{H}_{1}$ characteristic with the characteristic of the pressure pipeline $\mathrm{Q}-\mathrm{H}_{\mathrm{PP} 1}$ with flow $\mathrm{Q}_{1}$. As a result of an increase in the level of the tailwater in the intake structure of the PS by the value $\Delta \mathrm{h}$, its static head $\mathrm{H}_{\mathrm{SP} 1}$ will increase by the same value $\Delta \mathrm{h}$ and took a position equal to $\mathrm{H}_{\mathrm{SP} 2}=\mathrm{H}_{\mathrm{SP} 1}-\Delta \mathrm{h}$.

Consequently, due to a change in the static component of the head $\mathrm{H}_{\mathrm{SP} 2}$, the characteristic of the pressure pipeline Q- $\mathrm{H}_{\mathrm{PP} 2}$ will take some new position with an intermediate point $B$ on the flow-pressure characteristic $\mathrm{Q}_{1}-\mathrm{H}_{1}$. However, this new position of the intermediate point $B$ in the operation of the pumping unit with the corresponding supply $\mathrm{Q}_{2}$ is not able to fully compensate for the amount of water entering the forward chamber of the water intake structure of the PS when its level rises. In this case, there is a mismatch of the reference signal of the water level control system with the signal coming from the water level sensor installed in the water intake structure of the PS. As a result, the regulator issues a signal processed according to the PI-law to the control system of the frequency-controlled electric drive, which contributes to an increase in the angular speed (rotation frequency) of the drive motor $\mathrm{M}$ of the pump unit (Fig. 2). At the same time, the flow-pressure characteristic of the centrifugal pump $\mathrm{Q}_{2}-\mathrm{H}_{2}$, corresponding to the angular velocity $\omega_{2}$, at the point of intersection with the characteristic of the pressure pipeline Q-HP2 forms a new steady-state operating point $\mathrm{C}$ (Fig. 3, a), with the supply of $\mathrm{Q}_{3}$ and contributing to the establishment of a predetermined level of the water head in the front chamber of the water intake structure of the PS.
With a slight decrease in the inflow of water in lowwater periods into the intake structure of the PS, the control system, contributing to the stabilization of the pool level, acts similarly to the above, but in the opposite direction (Fig. 3, b). As a result, the control system contributes to maintaining a given water level both in the diversion canal and in the water intake structure of the PS, ensuring the equality of the pump supply and water inflow, which realizes the process of stabilizing the level of the water horizon in the lower pool of the PS. Thus, ensuring the operation of the pumping unit in an economical energy-resource-saving mode is achieved.

A change in the angular speed of the pump impeller leads to a change in all of its operating parameters. This also changes the operating characteristic of the pump. Recalculation of pump characteristics for a different angular velocity is carried out using the so-called reduction formulas [8]

serving $\quad \frac{Q_{1}}{Q_{2}}=\frac{\omega_{1}}{\omega_{2}} ;$

for pressure $\quad \frac{\mathrm{H}_{1}}{\mathrm{H}_{2}}=\left(\frac{\omega_{1}}{\omega_{2}}\right)^{2}$;

for power

$$
\frac{\mathrm{P}_{1}}{\mathrm{P}_{2}} \approx\left(\frac{\omega_{1}}{\omega_{2}}\right)^{3}
$$

for the moment of static resistance $\frac{\mathrm{M}_{1}}{\mathrm{M}_{2}} \approx\left(\frac{\omega_{1}}{\omega_{2}}\right)^{2}$

Equality in formulas (3) and (4) is approximately, since when the flow rate $\mathrm{Q}$ and the head $\mathrm{H}$ change, the efficiency changes simultaneously.

The flow-pressure characteristic of a pump operating with a variable angular velocity of its impeller is described according to the equation of a quadratic parabola:

$$
H=H_{F} *\left(\frac{\omega}{\omega_{\text {nom }}}\right)^{2}-R_{F} * Q^{2},
$$

where $\mathrm{H}_{\mathrm{F}}$ - is a fictitious head corresponding to zero pump flow, in the calculations it should be taken: for pure water pumps $\mathrm{H}_{\mathrm{F}}=1.25 * \mathrm{H}_{\text {nom; }}$; for dynamic and dredge pumps $\mathrm{H}_{\mathrm{F}}=1.45 * \mathrm{H}_{\text {nom }} ; \mathrm{H}_{\text {nom }}-$ nominal (passport) pump head; $\omega, \omega_{\text {nom }}$ - variable and nominal angular velocity of the pump, respectively; $\mathrm{R}_{\mathrm{F}}$ - hydraulic fictitious resistance of the pump.

The characteristic of the pressure pipeline is described by the relationship [4] 


$$
\mathrm{H}=\mathrm{H}_{S P}+R * Q^{2} \text {. }
$$

The joint solution of the equation of the flow-pressure characteristics of the pump (5) with the equation of the pressure pipeline (6) with respect to the flow Q, allows us to obtain the dependence of the change in the pump flow on its angular velocity:

$$
Q=Q_{n o m} * \sqrt{\frac{\mathrm{H}_{F} *\left(\frac{\omega}{\omega_{n o m}}\right)^{2}-\mathrm{H}_{P P}}{\mathrm{H}_{F}-\mathrm{H}_{P P}}},
$$

where $\mathrm{Q}_{\text {nom }}$ - nominal (passport) pump flow; $\mathrm{H}_{\mathrm{SP}}$ - the static head of the pump, is defined as the difference between the geodetic marks of the highest point of fluid supply and the place where the pump is installed.

The joint solution of the equation of the flow-pressure characteristics of the pump (5) and the equation of the pressure pipeline (6) with respect to the head $\mathrm{H}$, allows obtaining the dependence of the change in the pump head on its angular velocity:

$$
\mathrm{H}=\mathrm{H}_{S P}+\left(\mathrm{H}_{n o m}-\mathrm{H}_{S P}\right) * \frac{\mathrm{H}_{F} *\left(\frac{\omega}{\omega_{n o m}}\right)^{2}-\mathrm{H}_{S P}}{\mathrm{H}_{F}-\mathrm{H}_{S P}},
$$

Dependence (8) is valid if the pump is selected correctly, and the pipeline is designed so that at the rated speed the rated flow and head are provided.

The most important parameter of a pump is its efficiency. The change in efficiency depending on the angular speed of the pump is determined using the Moody formula, converted for pumps [8]:

$$
\eta_{\text {pump }}=1-\frac{1-\eta_{\text {nom }}}{\left(\frac{\omega}{\omega_{\text {nom }}}\right)^{0,36}}
$$

where $\eta_{\text {nom }}$ is the nominal (passport) efficiency of the pump.

The power consumed by the pump is determined by the expression:

$$
\mathrm{P}_{\text {pump }}=\frac{\gamma * Q * H}{102 * \eta_{\text {pump }}}, \kappa \mathrm{W}
$$

where $\gamma$ is the density of the pumped liquid, $\mathrm{kg} / \mathrm{m} 3$ (for pure water $\gamma \approx 1000 \mathrm{~kg} / \mathrm{m} 3$ ); Q - second pump flow, $\mathrm{m} 3 / \mathrm{s} ; \mathrm{H}$ - pump head, m (meters of water column); $\eta_{\text {pump }}$ - pump efficiency, in relative units (p.u.).

The analysis of expression (10) for the power consumption of the pump $\mathrm{P}_{\text {pump }}$ shows that the real dependence of the power on the angular velocity differs more from the cubic parabola described by the reduction formula (3), the greater the value of the static head, and only with $\mathrm{H}_{\mathrm{SP}}=0$ the power consumed by the pump is proportional to the angular velocity in the cube.

A very important parameter of the pump is its mechanical characteristics, i.e. dependence of the moment of static resistance on the angular speed of the impeller. The energy indicators of the pump (losses and efficiency) depend on the type of mechanical characteristics. The moment of static resistance of any mechanism, including the pump, is determined by the expression $\mathrm{M}_{\mathrm{R}}=\mathrm{P} / \omega$, or

$$
\mathrm{M}_{\text {R.pump }}=\frac{\gamma * Q * H * 10^{3}}{102 * \omega * \eta_{\text {pump }}}, \mathrm{Nm}
$$

Analysis of expression (11) for the moment of static resistance of the pump $M_{\text {R.pump }}$ shows that the real dependence of the moment of static resistance on the angular velocity differs more from the quadratic parabola described by the reduction formula (4), the greater the value of the static head, and only at $\mathrm{H}_{\mathrm{SP}}=0$ the moment of static resistance is proportional to the angular velocity squared.

Equation (11) is valid until the head developed by the pump equals the static head. From this time on, the pump flow and its efficiency become equal to zero, which corresponds to the boundary angular velocity according to (8)

$$
\omega_{B}=\omega_{\text {nom }} * \sqrt{\mathrm{H}_{S P} / \mathrm{H}_{F}} .
$$

The calculation of the static head $\mathrm{H}_{\mathrm{SP}}$ (stabilized value), taking into account the characteristics of the pressure pipeline, is carried out according to the following formula

$$
\mathrm{H}_{S P}=\frac{\mathrm{H}-\mathrm{H}_{\text {nom }} *\left(\frac{Q}{Q \text { nom }}\right)^{2}}{1-Q_{\text {in }} *\left(\frac{Q}{Q \text { nom }}\right)^{2}}
$$

where $\mathrm{Q}_{\text {in }}$ - the amount of water (its inflow) into the intake structure of the pumping station station.

The water level is calculated using the formula

$$
\mathrm{H}_{L}=\mathrm{H}_{h}-\mathrm{H}_{S P} \text {, }
$$

where $\mathrm{H}_{\mathrm{h}}$ is the geodetic height of water rise.

The height of water rise is determined by the difference in geodetic marks between the highest point of its rise and the place where the pump is installed, and the static head is determined by the difference in geodetic marks between the highest point of water rise and the level in the supply channel or intake structure of the pumping station.

Formula (14) can be used to select the type of level sensor. Considering that the pumped medium is water, it is possible to recommend a submersible probe for measuring the liquid level of the "Metran" type, which has the following technical characteristics and parameters:

$>$ measured medium - non-aggressive liquids and viscous media;

$>$ pressure (level) measurement range: $4 \mathrm{kPa} \div 1 \mathrm{mPa}$ $(04 \div 100 \mathrm{~m} \mathrm{wc})$

$>$ probe diameter $45 \mathrm{~mm}$;

$>$ output signal $4 \div 20 \mathrm{~mA}, 0 \div 10 \mathrm{~V}$.

models of magnetic circuits developed by MFS.

\section{Block diagram of the drive motor of the pump unit with frequency control with stabilization of the water level}

The structural diagram of the drive motor of the pump unit with frequency control with stabilization of the water level is presented in the form of typical dynamic links. The structural diagram of the pump is presented in the form of functional blocks that implement various types of dependences on flow, pressure, power and moment of static resistance.

The block diagram of a frequency-controlled pumping unit with stabilization of the water level in the intake structure or the derivation channel of the pumping station is shown in (Fig. 4).

The blocks of the structural diagram of the pumping unit are the functional dependences of the flow $Q=f(\omega)$ (7), the head $H=f(\omega)(9)$, the power $P_{\text {pump }}=f(Q, H)(11)$ 
and the moment of the static resistance of the $M_{R \cdot p u m p}=f$ (w) (14) from the angular velocity of the pump.

The synthesis of a single-loop water level stabilization system is performed in the following sequence:

$>$ according to the data of the Office of Pumping Stations (UNS), the smallest inflow of water $Q_{\text {in }}$ in the planned period of time is determined;

$>$ for the smallest water inflow, the required angular velocity of the pump unit is determined in accordance with expression (12);

$>$ according to dependence (9), the possibility of the pump functioning in the working area is checked;

$>$ according to the technical installation data of the pumping unit, the minimum height of the static head $\mathrm{H}_{\text {SP.min }}$ is determined, which makes it possible to obtain the maximum possible water level $\mathrm{H}_{\text {L.max }}$ in the derivation channel;

$>$ when determining the gain of the level feedback, they are guided by the following considerations, namely the control signal

is reduced by half compared to the nominal value for the possibility of creating a stabilized level both higher and lower relative to the required optimal setting, which in practice provides a more flexible adjustment of the automatic stabilization system.

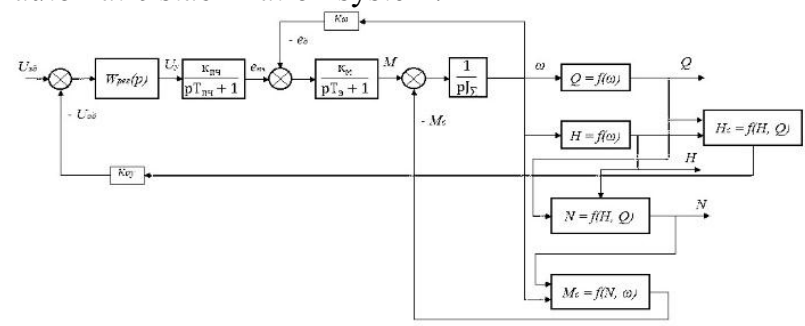

Fig. 4. Block diagram of a frequency-controlled pumping unit with stabilization of the water level in the intake structure of the pumping station

In order to analyze the effect of changes in the levels of water horizons in the water intake structure of the PS on the energy- and resource-saving modes of operation of the PU, we use a specific example of the PS Ramadan of the Tashkent region, taking into account the actual technical characteristics of hydromechanical and electromechanical equipment, taking into account the centrifugal pump of the 5000D-32 brand, drive asynchronous electric motor type A4-450 U-8UZ, as well as geometric dimensions along the entire length of the pressure network, including the suction, communication, supply and common pressure pipelines, as well as the values of head losses in local resistances (pressure valves, check valves, places of smooth rotation, gradual expansion or contraction, etc.), in accordance with the developed mathematical model and methodology, the main operating parameters of the pumping station Ramadan were calculated, which are shown in Table 1.

In fig. 5 shows the dependences of the change in the supply, pressure, efficiency, power consumption and specific power consumption of the PU on the change in the horizon of the water surface in the water intake structure of the PS, i.e., the increase in $\mathrm{H}_{\mathrm{SP}}$, expressed in relative units $\left(Q^{*}=Q / Q_{\mathrm{H}}, \mathrm{H}^{*}=\mathrm{H} / \mathrm{H}_{\mathrm{H}}, \eta^{*}=\eta / \eta_{\text {pump }} *\right.$ $\left.\eta_{\text {motor }}, \mathrm{P}^{*}=\mathrm{P} / \mathrm{P}_{\mathrm{H}}, \Delta \ni^{*}=\Delta \ni / \Delta Э_{\mathrm{T}}\right)$, where $\Delta Э_{\mathrm{T}}-$ is the theoretical specific power consumption of the supplied water PU, allowing to determine the most rational range of stabilization and maintenance of the level of the lower pool of the PS

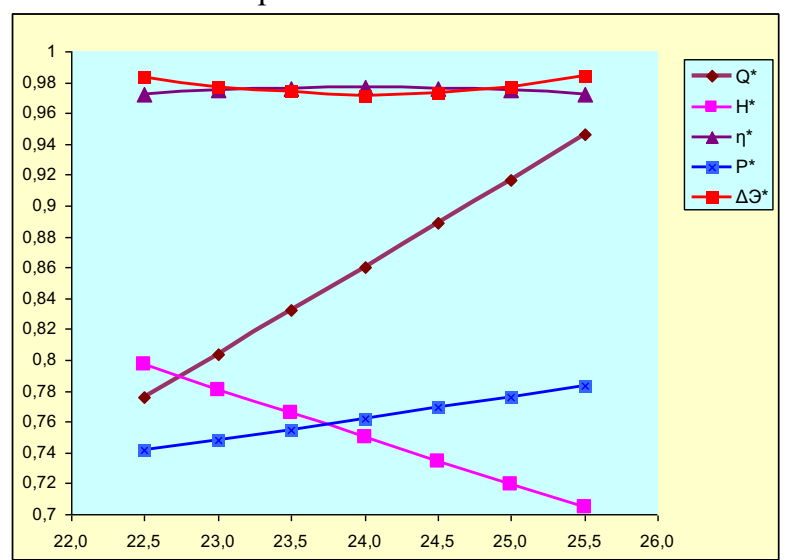

Fig. 5. The influence of changes in the level of the downstream of the pumping Ramadan station on the main parameters of its pumping unit

The graph (Fig. 5) shows that Ramadan is the most preferred for PS the level in the downstream of the water intake structure of the pumping station, where $\mathrm{H}_{\mathrm{SP}}=24$ $\mathrm{m}$, is the highest, at which the operation of the scientific equipment is ensured with the lowest specific power consumption. Along with this, it can be assumed that in the indicated vicinity of the change in the height of the lower level of the head of the PS $\nabla_{L} \approx \pm 0.25 \mathrm{~m}$, it can also practically be considered that it will correspond to the permissible range of stable maintenance of the level of the head of the PS.

Obviously, based on the actual operational and technical characteristics of hydromechanical and electromechanical equipment, for each PS, the optimal and minimum permissible ranges of the fluctuation levels of the wells should be determined in order to ensure energy and resource-saving modes of operation of the PU.

Based on the foregoing, it should be considered indisputable that ensuring the efficient operation of the PU and the achievement of the desired values of the parameters of their hydromechanical equipment can also be obtained by controlling the $\mathrm{H}_{\mathrm{SP}}$ by stabilizing and maintaining the levels of the wells within the specified required limits, as well as by properly regulating the supply of the PU, therefore water supply to PS in order to maximize the alignment of water consumption schedules and their coverage. This can be realized with a certain combination of the number of functioning PS (step regulation), in combination with smooth regulation of the supply of one of them, which will make it possible to satisfy the most required mode of water supply of PS with improved energy indicators. 
Table 1. The results of calculating the parameters of the pumping unit when the level changes the downstream of the intake structure of the Ramadan pumping station

\begin{tabular}{|c|c|c|c|c|c|c|}
\hline № & $\begin{array}{c}\text { Difference levels } \\
\text { beefs } \mathrm{H}_{S P}^{*}, \mathrm{~m}\end{array}$ & $\begin{array}{c}\text { Innings PU } \\
\mathrm{Q}^{*}, \mathrm{~m}^{3} / \mathrm{s}\end{array}$ & $\begin{array}{c}\text { Pressure PU } \\
\mathrm{H}^{*}, \mathrm{~m}\end{array}$ & $\begin{array}{l}\text { Efficiency } \\
\text { PU } \eta^{*}\end{array}$ & $\begin{array}{c}\text { Power } \\
\text { PU P* } P^{*}, \mathrm{~kW}\end{array}$ & $\begin{array}{c}\text { Specific consumption of } \\
\text { electricity PU } \Delta \ni^{*}, \mathrm{~kW} * \\
p h / \mathrm{mln} . \mathrm{m}^{3} * m\end{array}$ \\
\hline 1. & 22,5 & 0,776 & 0,797 & 0,972 & 0,742 & 0,9836 \\
\hline 2. & 23 & 0,804 & 0,781 & 0,975 & 0,748 & 0,9768 \\
\hline 3. & 23,5 & 0,832 & 0,766 & 0,976 & 0,755 & 0,9742 \\
\hline 4. & 24 & 0,860 & 0,750 & 0,977 & 0,762 & 0,9711 \\
\hline 5. & 24,5 & 0,889 & 0,734 & 0,976 & 0,769 & 0,9729 \\
\hline 6. & 25 & 0,917 & 0,719 & 0,975 & 0,776 & 0,9765 \\
\hline 7. & 25,5 & 0,946 & 0,705 & 0,972 & 0,783 & 0,9837 \\
\hline
\end{tabular}

\section{Conclusion}

1.The proposed graphical - analytical method for calculating the parameters of a pumping unit with frequency regulation of its performance makes it possible to obtain the corresponding pressure - flow characteristic of the pump.

2. The investigated features of the process of stabilization of the water level with frequency control make it possible to determine the most rational levels of the water surface in the front chamber of the intake structure of the pumping station, contributing to the achievement of the minimum value of the specific power consumption.

3. The proposed block diagram of a frequency-controlled pumping unit with frequency control with level stabilization in the form of typical dynamic links makes it possible to implement various types of functional dependences of the flow $Q=f(\omega)$, head $H=f(\omega)$, power $P_{\text {pump }}=f(Q, H)$ and the moment of static resistance of the $M_{\text {R.pump }}=f(\omega)$ from the angular speed of the pump.

\section{References}

1. Allaev K. R. Energy of the world and Uzbekistan. - T.: "Molia", 2007, 388 p. (in Uzbekistan).

2. Anikin Yu. V., Tsarev N. S., Ushakova L. I. Pumps and pumping stations. - Yekaterinburg: Ural publishing House. UN-T., 2018. - 138 p. (in Russian).

3. Khamudkhanov M. M., Abdullabekov I. A., Khamudkhanova N. B. The control device of the pump station. Application for invention IAP 20180529 (Republic of Uzbekistan). Official Bulletin. - Tashkent, 2018. - No. 12. Pp. 33-34. (in Uzbekistan).

4. Khamudkhanov M M 2013 Pumping station of pump irrigation as an object of energy and resource saving control Uzbek journal «Bulletin of TashSTU» № 2 pp 67 -73 .

5. Kamalov T. S. Frequency-regulated electric drive of pumping stations of machine irrigation systems. T.: Fan, 2014, 354 p. (in Uzbekistan).

6. Kadirov A.A., Khamudkhanov M.M. Methods of research and calculation of energy-resource-saving operating modes of water-lifting pumping stations. -T: "Yangi asr avlodi", 2013. -120 p. (in Uzbekistan).

7. Leznov B. S. Frequency-adjustable electric drive of pumping units. - Moscow: Mashinostroenie, 2013. - 176 p. (in Russian).

8. Khamudkhanov M. M., Abdullabekov I. A., Khamudkhanova N. B. Controls of the modes of operation of the pumping station with application of frequencycontrolled electric drive. Engineering-2020 IOP Conf. Series: Materials Science and Engineering 862 (2020)

9. Automated electric drive / ed. N.F. Ilyinsky and M.G. Yunkova. - M.: Energoatomizdat, 1990, -544 p. (in Russian).

10. Noll P. Determining the real cost of powering a pump. World Pumps. 2008. 1ss. 496. P. 32-34.

11. Zakhidov R. A. Energy saving management in developed countries. Problems of energy and resource saving. - Tashkent, 2005. - No. 1. Pp. 114-121. (in Uzbekistan). 